\title{
Spectroscopic analysis of the nuclear and circumnuclear regions of the Seyfert 2 galaxy NGC 7130 *
}

\author{
M. Radovich, P. Rafanelli, Padova, Italy \\ Department of Astronomy, University of Padova \\ K. Birkle, Heidelberg, Germany \\ Max-Planck-Institut für Astronomie \\ G.M. Richter, Potsdam, Germany \\ Astrophysikalisches Institut Potsdam
}

Received 1997 May 24; accepted 1997 July 2

\begin{abstract}
We discuss here the spectroscopic properties of the Seyfert 2 galaxy NGC 7130 (= IC 5135). Emission line regions were isolated and line ratios were measured and fitted with photoionization models; this allowed to discriminate between thermal and non thermal ionization in the circumnuclear regions. Massive star formation is likely to occur at projected distances from the nucleus $\geq 2 \mathrm{kpc}$ : line profiles in these regions suggest the presence of outflows of gas due to stellar winds from hot massive stars.
\end{abstract}

Key words: active galaxies: Seyfert galaxy: NGC 7130 - spectroscopic properies

AAA subject classification: 158

1.

Introduction

NGC 7130 ( $\mathrm{z}=0.016$, morphological type Sa pec) was classified as a Sy 2 by Phillips, Charles and Baldwin (1983). Ultraviolet spectra obtained with IUE in a $10^{\prime \prime} \times 20^{\prime \prime}$ aperture showed (Thuan 1984) the presence of blueshifted CIV $\lambda 1549$ and SiIV $\lambda 1400$ absorption lines, which are likely due to stellar winds associated with hot and massive O stars. Shields and Filippenko (1990, SF hereafter) analyzed the inner $6^{\prime \prime}$ with long-slit spectroscopy at a position angle (P.A.) of $11^{\circ}$ along the galaxy: they concluded that a transition is present between non thermal and thermal ionization from the inner to the outer regions. They also obtained a high resolution $(1.8 \AA)$ spectrum in a $2^{\prime \prime} \times 4^{\prime \prime}$ region centered on the nucleus at P.A. $=90^{\circ}$ and found that two components with line widths (FWHM) of $\sim 1000$ and $250 \mathrm{~km} \mathrm{~s}^{-1}$ are present in the profiles of both forbidden and permitted lines, which they interpreted as the kinematical counterparts to the two components identified in the UV by Thuan (1984): namely, an active nucleus from which arise the broad components of the emission lines and circumnuclear HII regions of high-metallicity that produce the narrow cores. NGC 7130 is also a powerful infrared source, $L_{60-100 \mu m}=4.3 \times 10^{44} \mathrm{erg} \mathrm{s}^{-1}$ (Lonsdale 1985): far infrared emission is likely powered by bursts of star formation in Hll regions surrounding the Seyfert nucleus, as confirmed by the spectral index $\alpha(60,25) \sim-2.3$, steeper than in a typical Seyfert, $\alpha(60,25)>-1.5$.

2.

Observations and reductions

Two long-slit spectra were taken at LaSilla on November 1996 with the ESO 2.2m telescope + EFOSCII and a $2^{\prime \prime}$ wide long slit oriented at position angles $90^{\circ}$ and $160^{\circ}$ along the galaxy; a spectral resolution of $10 \AA$ at $5700 \AA$ was measured from the FWHM of a faint comparison line. The linear scale along the slit is $0.31 \mathrm{kpc} /{ }^{\prime \prime}(\mathrm{z}=0.016$, $\mathrm{H}=75 \mathrm{~km} \mathrm{~s}^{-1} \mathrm{Mpc}^{-1}$ ).

"Poster presented at the Asiago Meeting "Environmental and physical properties of nearby AGN" on 23-24 April, 1997 

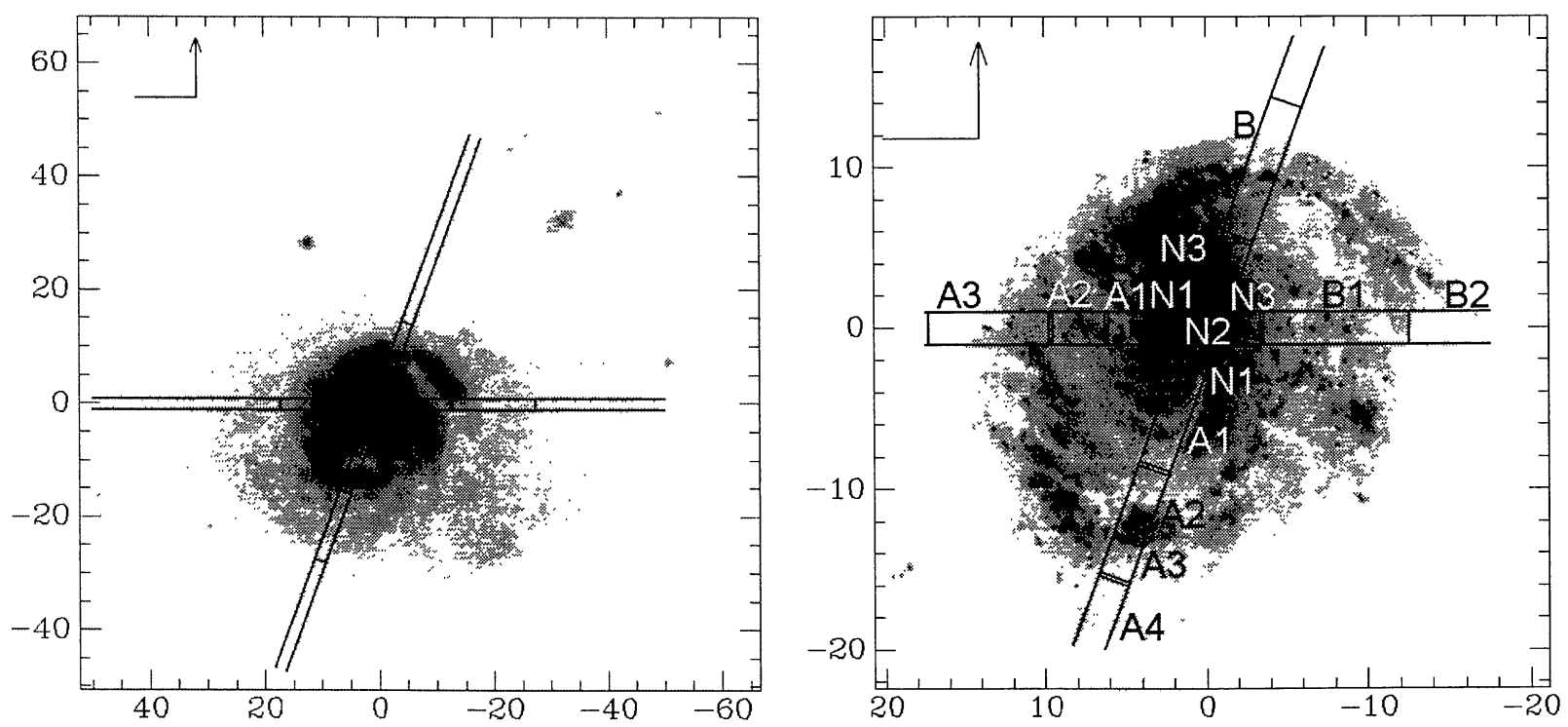

Fig. 1. NGC 7130 imaged through: left - the R filter with EFOSC2 at the 2.2m; right - the HST WFPC2 camera (F606W filter). The positions of the slit (P.A. $=90^{\circ}, 160^{\circ}$ ) and the regions extracted are shown for comparison; north is at the top, east at the left.

The usual calibration steps were applied to the 2D spectra (bias subtraction, flat field correction, wavelength and flux calibration, atmospheric extinction correction, background subtraction): to this aim we used the LONGSLIT package in the NOAO's imaging analysis software IRAF. 1D spectra were then extracted according to the $\mathrm{H} \alpha$ and $\mathrm{H} \beta$ emission features in $2 \mathrm{D}$ contour maps.

In the nuclear regions, narrow and broad components were deblended following the procedure the details of which will be given below.

In the circumnuclear regions, we had to subtract the absorption line spectrum of the underlying galaxy in order to have a reliable estimate of the Balmer emission line fluxes: this was done using as a template the spectrum of an early type galaxy without emission lines observed with similar spectral resolution and in the same spectral range. Both the template and the object spectra were shifted to the rest wavelength frame measuring the centroids of different absorption lines.

After that, we plotted the so-called Veilleux-Osterbrock diagrams (Veilleux and Osterbrock 1987, VO hereafter), namely [OIII] $\lambda 5007 / \mathrm{H} \beta$ vs. [NII] $\lambda 6583 / \mathrm{II} \alpha$, [SII] $(6716+6731) / \mathrm{H} \alpha$ and [OI] $\lambda 6300 / \mathrm{H} \alpha$, and computed a grid of photoionization models using the latest release of G. Ferland's code, Cloudy 90 (Ferland 1996). They are shown in Fig. .

An image of NGC 7130 was also taken in the same observing run using the R filter $\left(\lambda_{c}=6439 \AA\right.$, FWHM $=$ $1675 \AA$ ) and is shown in Fig. left after the division by the flat field. A much more detailed view of the structure of the spiral arms may be seen (Fig. right) in the image which was taken with the HST WFPC2 camera as a part of a snapshot survey of nearby AGNs (Gorjan 1995), using the F606W filter $\left(\lambda_{c}=5940 \AA, \Delta \lambda=1500 \AA\right.$ ) and reduced using the adaptive filtering procedures described by Lorenz et al. (1993). The emission in the arms around the nucleus is clearly resolved in bright knots which are likely to be HII regions.

3.

Results

3.1.

Nucleus

Three spectra $\sim 2^{\prime \prime}$ wide were extracted in the nuclear region $(\mathrm{N} 1, \mathrm{~N} 2, \mathrm{~N} 3)$. As mentioned above, line profiles in the inner $2^{\prime \prime}$ (N2) are characterized (Fig. and Fig.) by the presence of a broad component (FWHM $\sim 1000 \mathrm{~km}$ $\mathrm{s}^{-1}$ ) and a narrow core (FWHM $\sim 200 \mathrm{~km} \mathrm{~s}^{-1}$ ).

In order to measure the line fluxes (broad+narrow component) and build the VO diagrams (Fig. ), it was necessary to deblend $\mathrm{H} \alpha$ from the [NII] lines. To this aim, we first tried to use the profiles of the $\mathrm{H} \beta$ and [OIII] $\lambda 5007$ lines as templates, but large residuals were produced. We then followed the same procedure adopted by SF, namely we first built a synthetic line profile from the blue and red wings of the [SII] doublet: the resulting template profile was used to fit the components of the $\mathrm{H} \alpha+[\mathrm{NII}]$ complex, by interactively shifting in wavelength 

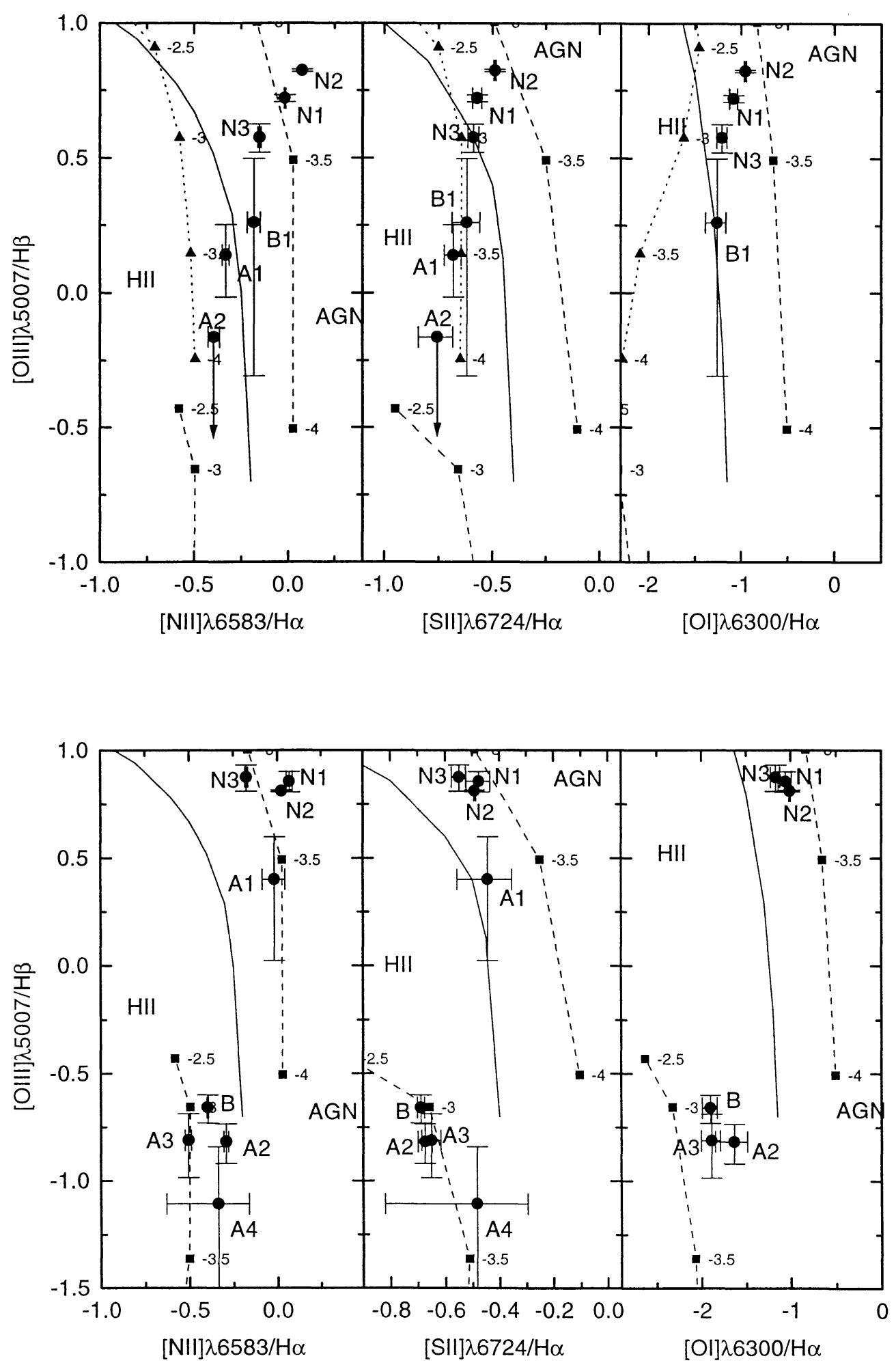

Fig. 2. VO line ratios for the nuclear and circumnuclear regions along P.A. $=90^{\circ}$ ( upper part) and P.A. $=160^{\circ}($ lower part $)$. Thermal (Mihalas continuum, $T=35000 \mathrm{~K}, \log n_{\mathrm{H}}=2$ ) and non thermal (power law, $\alpha=-1.5, \log n_{\mathrm{H}}=2$ ) models are also shown for different values of the ionization parameter; triangles in the upper panel show the ratios computed considering a combination of thermal $(\log U=-3)$ and non thermal ionization ( $\log U$ between -4 and -2.5 ). Note that 6724 means $6716+6731$. 
and scaling in intensity to minimize the residuals (Fig. upper part). In this way, we obtained in the nucleus line ratios (see Tab. ) which are in good agreement with the values given by SF. The VO line ratios are in agreement with those produced by a power-law continuum $\left(\alpha=-1.5, n_{\mathrm{H}}=10^{4} \mathrm{~cm}^{-3}, \log U \sim-2.7\right.$, solar abundances $)$.

iWe then tried to fit by multiple gaussians the $\mathrm{H} \beta$ and $[\mathrm{OIII}] \lambda 5007$ line profiles, as well as the synthetic line profiles of $\mathrm{H} \alpha$, [NII] $\lambda 6583$ and [SII] $\lambda \lambda 6716,6731$ obtained using the above mentioned procedure. All these lines are fitted by two gaussians with FWHM of $\sim 1000$ and $200 \mathrm{~km} \mathrm{~s}^{-1}$. In the case of the $\mathrm{H} \beta, \mathrm{H} \alpha,[\mathrm{NII}] \lambda \lambda 6548,6583$ and [SII] $\lambda \lambda 6716,6731$ lines the broad component is blueshifted $\left(\mathrm{cz} \sim 4660 \mathrm{~km} \mathrm{~s}^{-1}\right)$ if compared with the centroid of the narrow core (cz $\sim 4740 \mathrm{~km} \mathrm{~s}^{-1}$ ), whereas for other emission lines (e.g.: [OIII] $\lambda 5007$ ) the position of the centroid of the two components is nearly the same ( $\mathrm{cz} \sim 4740 \mathrm{~km} \mathrm{~s}^{-1}$ ), see Fig. .

Table 1. Emission line ratios measured in the nuclear regions. The uncertainties associated with the determination of the level of the continuum are given; values corrected for internal dust extinction are printed in bold. Fluxes are in units of $10^{-15} \mathrm{erg} / \mathrm{s} / \mathrm{cm}^{2}$.

\begin{tabular}{|c|c|c|c|c|c|c|c|}
\hline \multirow[b]{2}{*}{ Line } & \multirow[b]{2}{*}{$\lambda$} & \multicolumn{3}{|c|}{ P.A. $=90^{\circ}$} & \multicolumn{3}{|c|}{ P.A. $=160^{\circ}$} \\
\hline & & N1 & $\mathrm{N} 2$ & N3 & N1 & $\mathrm{N} 2$ & N3 \\
\hline [NeIII] & 3869 & $\begin{array}{c}1.05 \pm 0.05 \\
1.35\end{array}$ & $\begin{array}{c}0.52 \pm 0.02 \\
0.95\end{array}$ & $\begin{array}{c}\ldots \\
0.00\end{array}$ & $\begin{array}{c}2.10 \pm 0.42 \\
4.51\end{array}$ & $\begin{array}{c}0.79 \pm 0.02 \\
1.35\end{array}$ & $\begin{array}{c}2.00 \pm 0.34 \\
7.47\end{array}$ \\
\hline [NeIII] & 3968 & $\ldots$ & $\begin{array}{c}0.07 \pm 0.01 \\
0.12\end{array}$ & $\ldots$ & $\ldots$ & $\begin{array}{c}0.16 \pm 0.01 \\
0.26\end{array}$ & 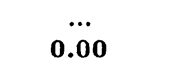 \\
\hline [SII] & 4071 & 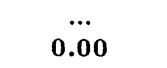 & $\begin{array}{c}0.17 \pm 0.02 \\
0.28\end{array}$ & $\ldots$ & $\ldots$ & $\begin{array}{c}0.32 \pm 0.02 \\
0.49\end{array}$ & $\ldots$ \\
\hline $\mathrm{H} \delta$ & 4102 & $\ldots$ & $\begin{array}{c}0.09 \pm 0.01 \\
0.14\end{array}$ & $\ldots$ & $\ldots$ & $\begin{array}{c}0.16 \pm 0.01 \\
0.24\end{array}$ & $\ldots$ \\
\hline $\mathrm{H} \gamma$ & 4340 & $\begin{array}{c}0.23 \pm 0.02 \\
0.27\end{array}$ & $\begin{array}{c}0.28 \pm 0.01 \\
0.40\end{array}$ & 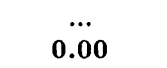 & $\ldots$ & $\begin{array}{c}0.33 \pm 0.01 \\
0.45\end{array}$ & $\begin{array}{c}\ldots \\
0.00\end{array}$ \\
\hline [OIII] & 4363 & $\begin{array}{c}0.03 \pm 0.01 \\
\mathbf{0 . 0 3}\end{array}$ & $\begin{array}{c}0.09 \pm 0.01 \\
0.13\end{array}$ & $\ldots$ & $\begin{array}{c}\ldots \\
0.00\end{array}$ & $\begin{array}{c}0.10 \pm 0.01 \\
0.14\end{array}$ & $\begin{array}{c}\ldots \\
0.00\end{array}$ \\
\hline HeII & 4686 & $\begin{array}{c}0.24 \pm 0.04 \\
0.25\end{array}$ & $\begin{array}{c}0.13 \pm 0.01 \\
0.15\end{array}$ & $\ldots$ & $\begin{array}{c}\ldots \\
0.00\end{array}$ & $\begin{array}{c}0.15 \pm 0.01 \\
0.17\end{array}$ & $\begin{array}{c}\ldots \\
0.00\end{array}$ \\
\hline [OIII] & 4959 & $\begin{array}{c}1.84 \pm 0.07 \\
1.80\end{array}$ & $\begin{array}{c}2.24 \pm 0.04 \\
2.13\end{array}$ & $\begin{array}{c}1.25 \pm 0.23 \\
1.14\end{array}$ & $\begin{array}{c}2.45 \pm 0.39 \\
2.30\end{array}$ & $\begin{array}{c}2.20 \pm 0.04 \\
2.11\end{array}$ & $\begin{array}{c}3.10 \pm 0.56 \\
2.78\end{array}$ \\
\hline [OIII] & 5007 & $\begin{array}{c}5.27 \pm 0.16 \\
\mathbf{5 . 1 0}\end{array}$ & $\begin{array}{c}6.68 \pm 0.07 \\
6.17\end{array}$ & $\begin{array}{c}3.76 \pm 0.45 \\
3.26\end{array}$ & $\begin{array}{c}7.16 \pm 0.79 \\
6.47\end{array}$ & $\begin{array}{c}6.49 \pm 0.06 \\
6.04\end{array}$ & $\begin{array}{c}7.51 \pm 1.05 \\
6.31\end{array}$ \\
\hline [NI] & 5199 & $\begin{array}{c}0.33 \pm 0.04 \\
0.30\end{array}$ & $\begin{array}{c}0.15 \pm 0.01 \\
0.12\end{array}$ & $\ldots$ & $\ldots$ & $\begin{array}{c}0.19 \pm 0.01 \\
0.16\end{array}$ & $\begin{array}{c}\ldots \\
0.00\end{array}$ \\
\hline $\mathrm{HeI}$ & 5876 & $\ldots .00$ & $\begin{array}{c}0.10 \pm 0.02 \\
0.06\end{array}$ & $\begin{array}{c}\ldots .00 \\
0.00\end{array}$ & $\ldots$ & $\begin{array}{c}0.12 \pm 0.01 \\
\mathbf{0 . 0 7}\end{array}$ & $\begin{array}{c}\ldots \\
0.00\end{array}$ \\
\hline [OI] & 6300 & $\begin{array}{c}0.33 \pm 0.04 \\
0.24\end{array}$ & $\begin{array}{c}0.70 \pm 0.03 \\
0.34\end{array}$ & $\begin{array}{c}0.78 \pm 0.16 \\
0.21\end{array}$ & $\begin{array}{c}0.70 \pm 0.17 \\
0.28\end{array}$ & $\begin{array}{c}0.57 \pm 0.02 \\
\mathbf{0 . 3 0}\end{array}$ & $\begin{array}{c}1.16 \pm 0.26 \\
0.24\end{array}$ \\
\hline [OI] & 6364 & $\ldots$ & $\begin{array}{c}0.28 \pm 0.03 \\
0.13\end{array}$ & $\ldots$ & $\ldots$ & $\begin{array}{c}0.23 \pm 0.02 \\
\mathbf{0 . 1 2}\end{array}$ & $\ldots$ \\
\hline [NII] & 6548 & $\begin{array}{c}1.22 \pm 0.02 \\
0.87\end{array}$ & $\begin{array}{c}1.85 \pm 0.02 \\
0.82\end{array}$ & $\begin{array}{c}1.88 \pm 0.23 \\
0.43\end{array}$ & $\begin{array}{c}3.05 \pm 0.40 \\
1.07\end{array}$ & $\begin{array}{c}1.93 \pm 0.02 \\
\mathbf{0 . 9 3}\end{array}$ & $\begin{array}{c}5.36 \pm 0.75 \\
0.89\end{array}$ \\
\hline $\mathrm{H} \alpha$ & 6563 & $\begin{array}{c}3.94 \pm 0.12 \\
\mathbf{2 . 8 0}\end{array}$ & $\begin{array}{c}6.38 \pm 0.06 \\
\mathbf{2 . 8 0}\end{array}$ & $\begin{array}{c}12.48 \pm 1.12 \\
2.80\end{array}$ & $\begin{array}{c}8.00 \pm 0.88 \\
2.80\end{array}$ & $\begin{array}{c}5.86 \pm 0.06 \\
\mathbf{2 . 8 0}\end{array}$ & $\begin{array}{c}17.10 \pm 2.05 \\
2.80\end{array}$ \\
\hline [NII] & 6583 & $\begin{array}{c}3.76 \pm 0.08 \\
2.66\end{array}$ & $\begin{array}{c}7.57 \pm 0.08 \\
3.30\end{array}$ & $\begin{array}{c}8.76 \pm 0.79 \\
1.94\end{array}$ & $\begin{array}{c}9.36 \pm 1.03 \\
3.25\end{array}$ & $\begin{array}{c}6.18 \pm 0.06 \\
2.93\end{array}$ & $\begin{array}{c}11.38 \pm 1.37 \\
1.83\end{array}$ \\
\hline [SII] & 6716 & $\begin{array}{c}0.61 \pm 0.01 \\
0.42\end{array}$ & $\begin{array}{c}0.97 \pm 0.01 \\
\mathbf{0 . 4 0}\end{array}$ & $\ldots$ & $\begin{array}{c}\ldots \\
0.00\end{array}$ & $\begin{array}{c}0.81 \pm 0.01 \\
\mathbf{0 . 3 7}\end{array}$ & $\begin{array}{l}\ldots .00 \\
0.0\end{array}$ \\
\hline [SII] & $6716+6731$ & $\begin{array}{c}1.06 \pm 0.06 \\
0.74\end{array}$ & $\begin{array}{c}2.07 \pm 0.04 \\
0.86\end{array}$ & $\begin{array}{c}3.23 \pm 0.42 \\
0.65\end{array}$ & $\begin{array}{c}2.68 \pm 0.46 \\
0.87\end{array}$ & $\begin{array}{c}1.89 \pm 0.04 \\
0.86\end{array}$ & $\begin{array}{c}4.82 \pm 0.77 \\
0.70\end{array}$ \\
\hline [SII] & 6731 & $\begin{array}{c}0.50 \pm 0.01 \\
0.35\end{array}$ & $\begin{array}{c}1.07 \pm 0.01 \\
0.44\end{array}$ & $\ldots$ & $\begin{array}{c}\ldots \\
0.00\end{array}$ & $\begin{array}{c}0.89 \pm 0.01 \\
0.40\end{array}$ & $\ldots$ \\
\hline [ArIII] & 7136 & $\begin{array}{c}\ldots \\
0.00\end{array}$ & $\begin{array}{c}0.20 \pm 0.01 \\
0.07\end{array}$ & $\ldots$ & $\begin{array}{c}\ldots .00 \\
0.00\end{array}$ & $\begin{array}{c}0.14 \pm 0.01 \\
0.06\end{array}$ & $\ldots$ \\
\hline [OII] & 7322 & $\begin{array}{c}0.46 \pm 0.08 \\
0.29 \\
\end{array}$ & $\begin{array}{c}0.40 \pm 0.02 \\
0.14 \\
\end{array}$ & $\begin{array}{l}\ldots \\
0.00\end{array}$ & $\begin{array}{c}\ldots \\
0.00 \\
\end{array}$ & $\begin{array}{c}0.35 \pm 0.02 \\
\mathbf{0 . 1 3} \\
\end{array}$ & $\begin{array}{c}\ldots \\
0.00 \\
\end{array}$ \\
\hline $\mathrm{F}(\mathrm{H} \alpha)$ & & $\begin{array}{c}40.9 \pm 0.4 \\
81.8\end{array}$ & $\begin{array}{c}256.9 \pm 0.0 \\
1367.0\end{array}$ & $\begin{array}{c}28.4 \pm 0.3 \\
589.3\end{array}$ & $\begin{array}{c}9.6 \pm 0.2 \\
81.1\end{array}$ & $\begin{array}{c}215.1 \pm 0.0 \\
963.0\end{array}$ & $\begin{array}{c}20.5 \pm 0.2 \\
806.7\end{array}$ \\
\hline $\mathrm{F}(\mathrm{H} \beta)$ & & $\begin{array}{c}10.4 \pm 0.2 \\
29.2\end{array}$ & $\begin{array}{c}40.3 \pm 0.4 \\
\mathbf{4 8 8 . 2}\end{array}$ & $\begin{array}{c}2.3 \pm 0.2 \\
210.5\end{array}$ & $\begin{array}{c}1.2 \pm 0.1 \\
28.9\end{array}$ & $\begin{array}{c}36.7 \pm 0.4 \\
\mathbf{3 4 4 . 1}\end{array}$ & $\begin{array}{c}1.2 \pm 0.1 \\
288.2\end{array}$ \\
\hline c & & 0.45 & 1.08 & 1.97 & 1.38 & 0.97 & 2.38 \\
\hline$E(B-V)$ & & 0.31 & 0.75 & 1.37 & 0.96 & 0.68 & 1.66 \\
\hline
\end{tabular}

The spectral resolution of our spectra, $\sim 10 \AA$, is too low for an unambiguous deblending of the line components. However, the observed nuclear line profiles and ratios are at least roughly consistent (Fig. lower part) with two populations of clouds as follows:

Broad component : $\log n_{\mathrm{H}} \sim 4, \log U \sim-2.7$ and an overabundance of nitrogen if compared with the solar value, $\mathrm{N} / \mathrm{N}_{\odot}=2$. 
Narrow component : $\log n_{\mathrm{H}} \sim 3, \log U \sim-3.5$ and solar abundances as defined in Ferland (1996).

Table 2. Emission line ratios measured in the circumnuclear regions; values corrected for internal dust extinction are printed in bold. Fluxes are given in units of $10^{-16} \mathrm{erg} / \mathrm{s} / \mathrm{cm}^{2}$.

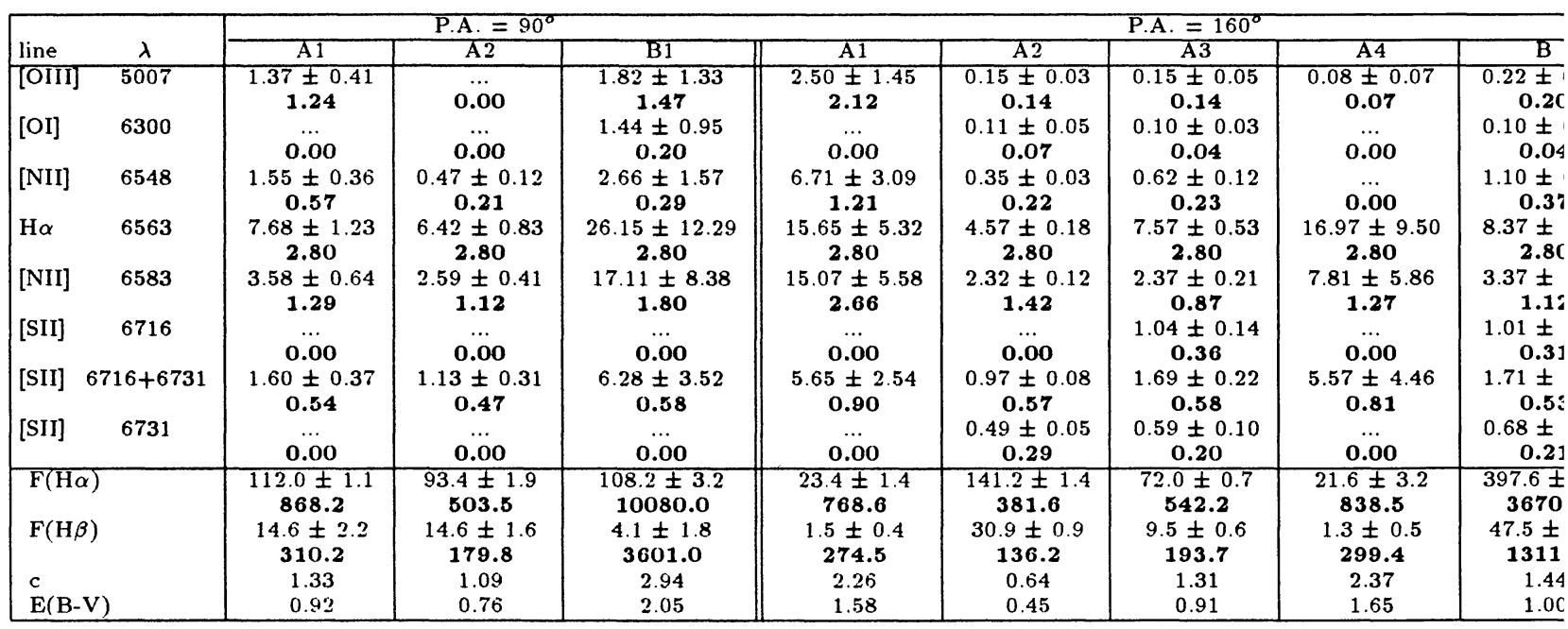
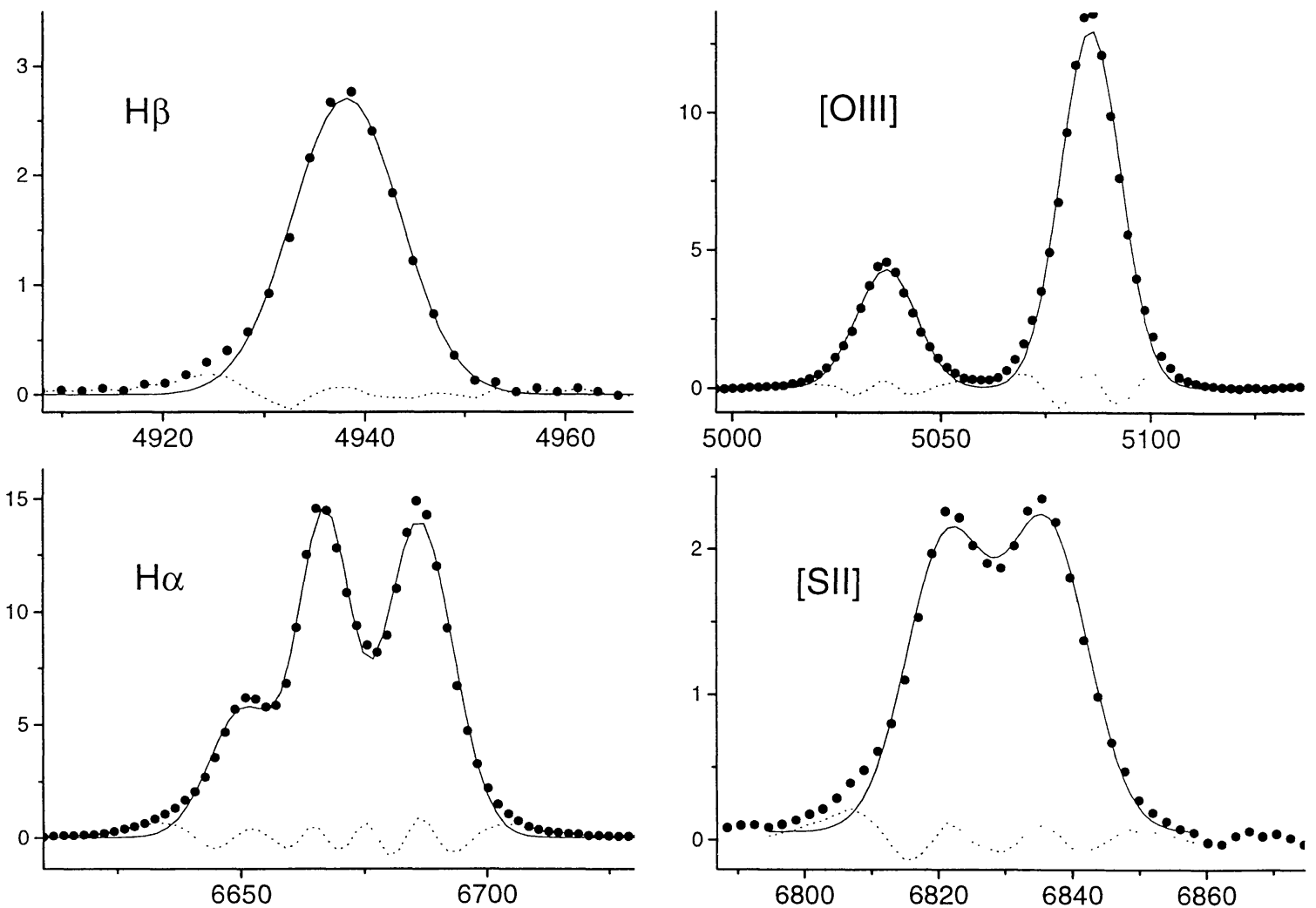

Fig. 3. Line profiles in the nucleus (N2) are compared with single-component gaussian fits: residuals in the blue wing are visible in $\mathrm{H} \beta,[\mathrm{NII}]+\mathrm{H} \alpha$, [SII] $\lambda \lambda 6716,6731$, but not in [OIII] $\lambda 5007$.

In both cases we assumed a power law continuum $\left(F_{\nu} \propto \nu^{\alpha}, \alpha=-1.5\right)$. We conclude that, even if circumnuclear massive star formation in the inner kpc of NGC 7130 can not be definitely ruled out, it is not required by the emission line properties. 

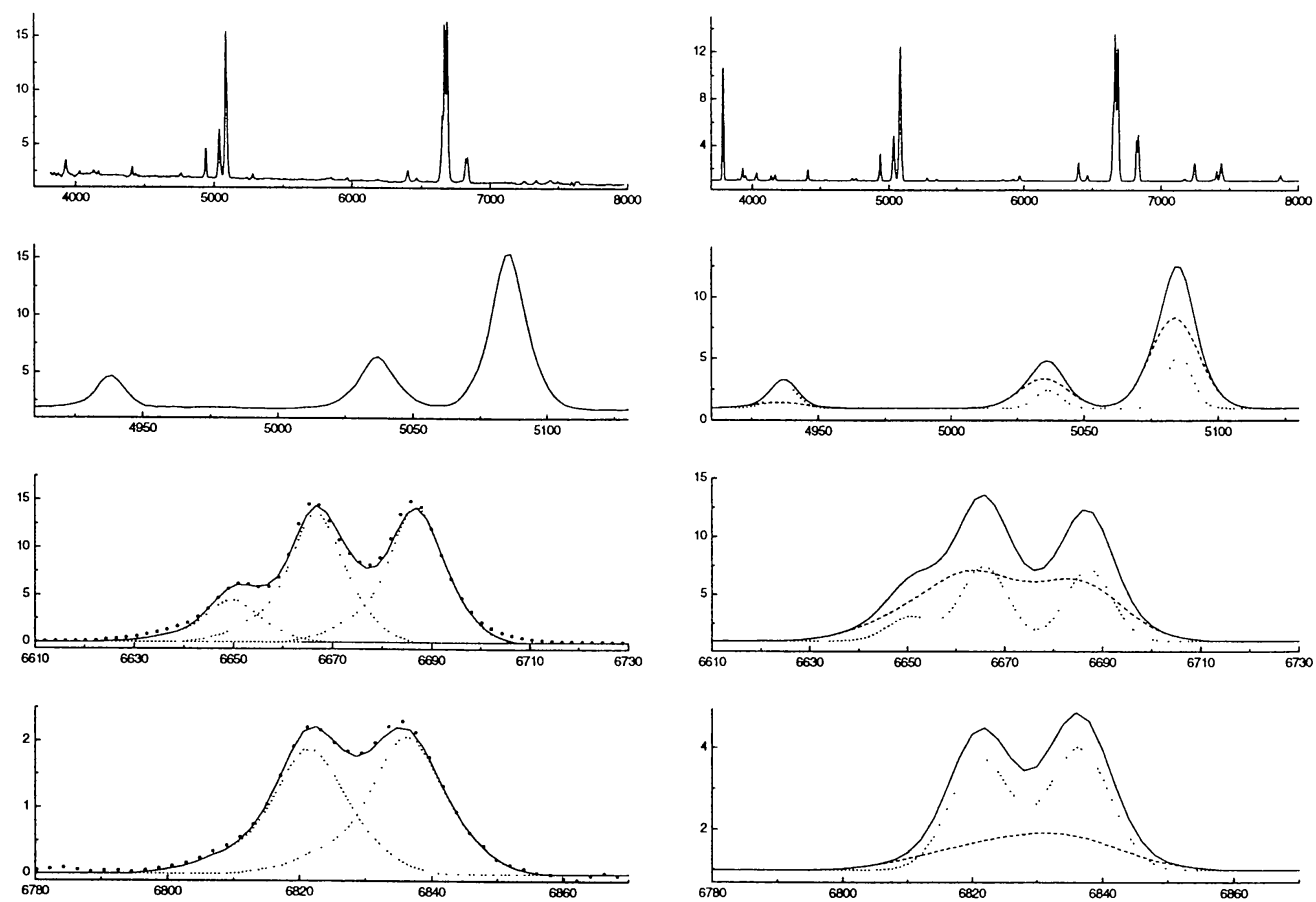

Fig. 4. Upper part: emission line profiles observed in the nucleus of NGC 7130 (region N2, 2" x 2" aperture, P.A. = $160^{\circ}$ ): the synthetic line profiles used in the deblending of [NII], $\mathrm{H} \alpha$ and [SII] lines are shown. Lower part: line fluxes obtained with Cloudy have been convolved with gaussians of FWHM $=1000 \mathrm{~km} \mathrm{~s}{ }^{-1}$ (dashed $\operatorname{line}: \log n_{\mathrm{H}}=4, \log U=-2.7, \mathrm{~N} / \mathrm{N}_{\odot}$ $=2, \mathrm{cz}=4600 \mathrm{~km} \mathrm{~s}^{-1}$ ) and $200 \mathrm{~km} \mathrm{~s}^{-1}$ (dotted line: $\log n_{\mathrm{H}}=3, \log U=-3.5$, solar abundances, $\mathrm{cz}=4700 \mathrm{~km} \mathrm{~s}$ ).

\subsection{Circumnuclear regions}

At a first look, emission lines in the circumnuclear regions are broad (FWIIM $\sim 500-600 \mathrm{~km} \mathrm{~s}^{-1}$ after the correction for the instrumental component), but a closer analysis (Fig. ) shows that the line profiles can not be fitted with simple gaussians: this is more evident in the spectra taken along $160^{\circ}$, where line profiles require at least two gaussians with nearly instrumental width: outflows of gas associated with massive star formation (Leitherer 1997) are likely to be present in these regions.

A complex kinematic is also evident from the observed radial velocities (Fig. ): the rotation curve along P.A. = $90^{\circ}$ is still reminiscent of a spiral rotation curve, whereas along P.A. $=160^{\circ}$ we see large deviations. The line ratios in the VO diagrams (Fig. , see also Tab. ) imply that the ionization is most likely thermal in the outer circumnuclear regions ( $\geq 2-3 \mathrm{kpc}$ ), namely in regions $\mathrm{A} 2, \mathrm{~A} 3, \mathrm{~A} 4$ and $\mathrm{B}$ along $160^{\circ}, \mathrm{A} 2$ along $90^{\circ}$ (but here the [OIII] $\lambda 5007 \mathrm{flux}$ is very uncertain). In these regions the line ratios shown in Fig. are best fitted by photoionization models with the following parameters: a non-LTE Mihalas stellar continuum (Mihalas 1972), $\log g=4$, with temperature $\mathrm{T}=$ $35000 \mathrm{~K}, \log n_{\mathrm{H}}=2$ and $\log U \sim-3$.

In the inner circumnuclear regions (A1 and B1) the ionization is likely given by a combination of thermal and
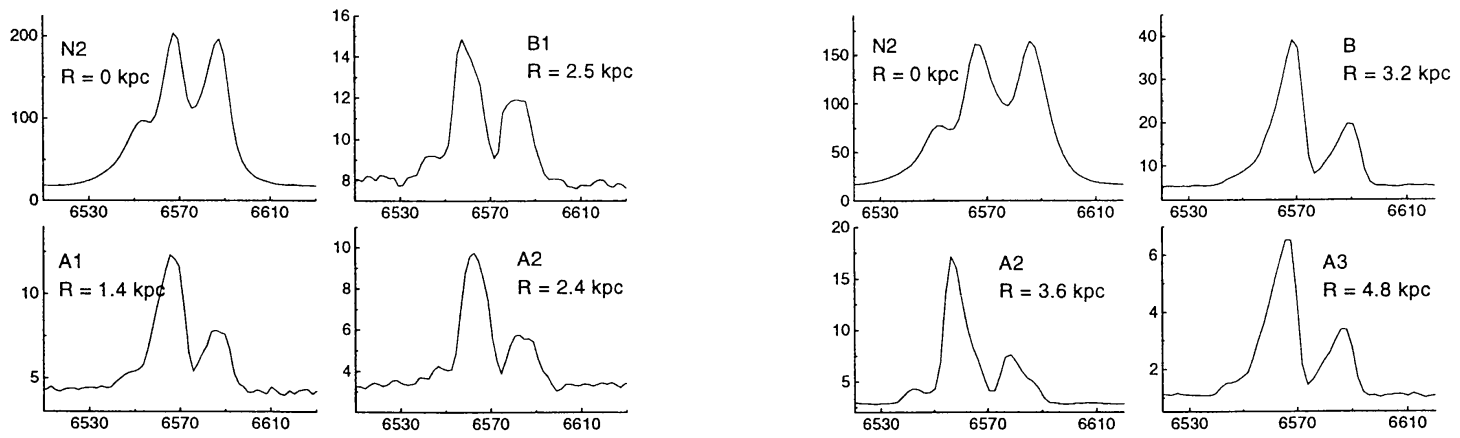

Fig. 5. $\mathrm{H} \alpha+[\mathrm{NII}]$ profiles in different regions along P.A. $=90^{\circ}($ left $)$ and P.A. $=160^{\circ}($ right). 

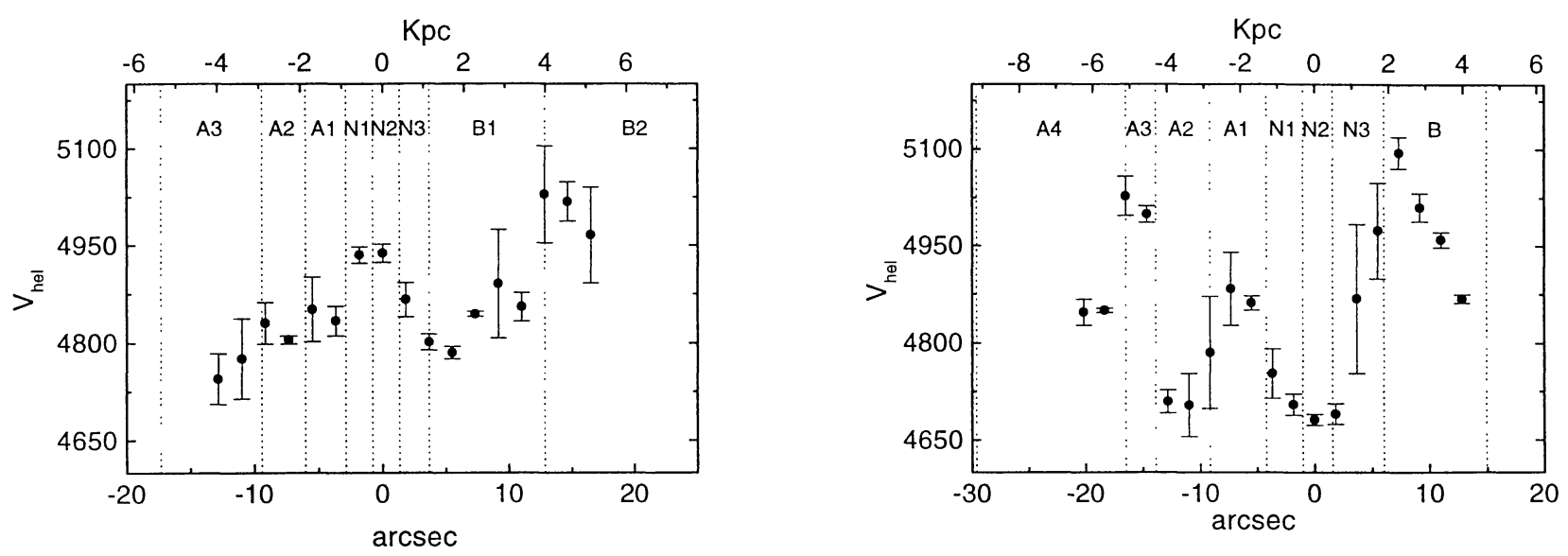

Fig. 6. Radial velocities computed from emission line centroids of $\mathrm{H} \alpha, \mathrm{H} \beta$, [OIII] $\lambda 5007$, [OI] $\lambda 6300$, [NII] $\lambda 6583$ versus the projected linear (top axis) and angular (bottom axis) distances from the nucleus, defined as the centroid of the continuum. Left: P.A $=90^{\circ} ;$ Right: P.A $=160^{\circ}$

diluted non thermal radiation: we tried to fit the line ratios with a model where a cloud of density $\log n_{\mathrm{H}}=2$ is ionized both by a thermal continuum with the parameters found above and a power law with a ionization parameter in the range $U=10^{-2.5}-10^{-4}$, namely for different distances from the nuclear source.

\section{Conclusions}

From the analysis of the emission lines of NGC 7130 we conclude that:

1. Massive star formation is not necessarily present in the inner $\mathrm{kpc}$, as instead proposed by SF from the analysis of the emission line profiles. Non thermal ionization may account for the observed intensities of both narrow and broad components: we have proposed a model where the narrow component, FWHM $200 \mathrm{~km} \mathrm{~s}^{-1}$, of the lines observed in the nuclear spectra is produced in clouds with density $n_{\mathrm{H}} \sim 10^{3} \mathrm{~cm}^{-3}$ and solar abundance, the broad component, FWHM $\sim 1000 \mathrm{~km} \mathrm{~s}^{-1}$, in clouds of higher density, $n_{\mathrm{H}} \geq 10^{4}$ $\mathrm{cm}^{-3}$ and an overabundance of nitrogen compared to the solar value. However, it must be noted that a more detailed analysis and more reliable modelling would require spectra with higher spectral and spatial resolution.

2. Massive star formation more likely occurs in the outer circumnuclear regions $(2-5 \mathrm{kpc})$. These regions are characterized by a complex kinematics, as seen from the observed radial velocities (Fig.), and by outflows of gas, as shown by the line profiles which are not fitted by simple gaussians (Fig. ). It is therefore likely that the P Cygni SiIV $\lambda 1400$ and CIV $\lambda 1549$ profiles visible in the IUE spectra (integrated over a $10^{\prime \prime} \times 20^{\prime \prime}$ aperture) shown by Thuan (1984) are produced in these regions rather than in the nucleus. Star formation activity in the circumnuclear regions is likely triggered by the interaction with the faint galaxy visible at NW in Fig. left. Between 1 and $2-3 \mathrm{kpc}$ the ionization is likely given by thermal radiation from stars and non thermal diluted radiation from the AGN.

Acknowledgements. We are grateful to Gary Ferland for having made available to the scientific community his photoionization code Cloudy 90. We acknowledge the access to the HST Science Data Archive and the ESO/ST-ECF Archive Facility, a joint collaboration of the European Space Observatory and the Space Telescope Coordinating Facility, for the retrieval of the HST WFPC2 image of NGC 7130.

\section{References}

Busko, I.C., and Steiner, J.E.: 1990, Mon. Not. R. Astron. Soc. 245, 470

Ferland, G.J.: 1996, Hazy, a brief introduction to Cloudy, University of Kentucky, Department of Physics and Astronomy Internal Report.

Gorjan V.: 1995, Astrophys. J. 450, L51

Leitherer, C.: 1997, Space Telescope Science Institute preprint n. 1123

$17^{*}$ 
Lonsdale, C.J.: 1985, Cataloged galaxies and quasars observed in the IRAS survey, Jet Propulsion Laboratory Lorenz, H., Richter, G. M., Capaccioli, M., and Longo, G.: 1993, Astron. Astrophys., 277, 321

Mihalas, D.: 1972, Non-LTE Model Atmospheres for B and O Stars (Boulder: NCAR) (NCAR-TN/STR-76)

Phillips, M.M., Charles, P.A., and Baldwin, J.A.: 1983, Astrophys. J. 266, 485

Shields, J.C., and Filippenko, A.V.: 1990, Astron. J. 100, 1034

Thuan, T.X.: 1984, Astrophys. J. 281, 126

Veilleux, S., and Osterbrock, D.E.: 1987, Astrophys. J. Suppl. Ser. 63, 311

Addresses of the authors:

M. Radovich, P. Rafanelli

Department of Astronomy

Vicolo dell'Osservatorio, 5

I-35122 Padova

Italy

K. Birkle

Max-Planck-Institut für Astronomie

Königstuhl

D-69117 Heidelberg

Germany

G. Richter Astrophysikalisches Institut Potsdam

An der Sternwarte, 16

D-14482 Potsdam

Germany 